\title{
On Fixed Point Theory of Monotone Mappings with Respect to a Partial Order Introduced by a Vector Functional in Cone Metric Spaces
}

\author{
Zhilong $\mathrm{Li}^{1}$ and Shujun Jiang ${ }^{2}$ \\ ${ }^{1}$ School of Statistics, Jiangxi University of Finance and Economics, Nanchang 330013, China \\ ${ }^{2}$ Department of Mathematics, Jiangxi University of Finance and Economics, Nanchang 330013, China \\ Correspondence should be addressed to Zhilong Li; lzl771218@sina.com
}

Received 19 November 2012; Revised 11 January 2013; Accepted 23 January 2013

Academic Editor: Micah Osilike

Copyright (C) 2013 Z. Li and S. Jiang. This is an open access article distributed under the Creative Commons Attribution License, which permits unrestricted use, distribution, and reproduction in any medium, provided the original work is properly cited.

We presented some maximal and minimal fixed point theorems of set-valued monotone mappings with respect to a partial order introduced by a vector functional in cone metric spaces. In addition, we proved not only the existence of maximal and minimal fixed points but also the existence of the largest and the least fixed points of single-valued increasing mappings. It is worth mentioning that the results on single-valued mappings in this paper are still new even in the case of metric spaces and hence they indeed improve the recent results.

\section{Introductions}

Throughout this paper, let $(X, d)$ be a complete cone metric space over a total minihedral and continuous cone $P$ of a normed vector space $E$. A vector functional $\varphi: X \rightarrow E$ introduces a partial order $\prec$ on $X$ as follows:

$$
x \prec y \Longleftrightarrow d(x, y) \preceq \varphi(x)-\varphi(y),
$$

for all $x, y \in X$, where $\preceq$ is the partial order on $E$ determined by the cone $P$. Using the partial order introduced by the vector functional $\varphi$, Agarwal and Khamsi [1] extended Caristi's fixed point theorem [2] to the case of cone metric space and proved that all mapping $T: X \rightarrow X$ (resp., $T: X \rightarrow 2^{X}$ ) such that

$$
\forall x \in X, \quad x \prec T x \text { (resp., } \forall x \in X, \exists y \in T x, x \prec y \text { ) }
$$

has a fixed point provided that $\varphi$ is lower semicontinuous and bounded below on $X$. In $[1,3]$, the authors studied Kirk's problem $[4,5]$ in the case of cone metric spaces and obtained some generalized Caristi's fixed point theorems in cone metric spaces. For the researches on the generalization of primitive Caristi's result in the case of metric spaces, we refer the readers to [6-12]. For other references concerned with various fixed point results for one, two, three, or four self-mappings in the setting of metric, ordered metric, partial metric, Prešić-type mappings, cone metric, G-metric spaces, and so forth, we refer the readers to [13-24].

In particular, when $E=\mathbb{R}$, the partial order defined by (1) is reduced to the one defined by Caristi [2] who denote it by $\prec_{1}$. Zhang $[25,26]$ and $\mathrm{Li}[27]$ considered the existence of fixed points of a mapping $T: X \rightarrow X$ (resp., $T: X \rightarrow 2^{X}$ ) such that

$$
\left.x_{0} \prec_{1} T x_{0} \quad \text { (resp., } \exists y \in T x_{0}, x_{0} \prec_{1} y\right)
$$

for some $x_{0} \in X$, and proved some maximal and minimal fixed point theorems at the expense that $T$ is monotone with respect to the partial order $\prec_{1}$.

In this paper, we shall extend the results of Zhang $[25,26]$ and $\mathrm{Li}$ [27] to the case of cone metric spaces. Some maximal and minimal fixed point theorems of set-valued monotone mappings with respect to the partial order $\prec$ are established in cone metric spaces. In addition, not only the existence of maximal and minimal fixed points but also the existence of largest and least fixed points is proved for single-valued increasing mappings. It is worth mentioning that the results 
on single-valued mappings in this paper are still new even in the case of metric spaces and hence they indeed improve the results of Zhang [25] and Li [27].

\section{Preliminaries}

First, we recall some definitions and properties of cones and cone metric spaces; these can be found in [1,3, 17-24, 28-30].

Let $E$ be a topological vector space. A cone $P$ of $E$ is a nonempty closed subset of $E$ such that $a x+b y \in P$ for all $x, y \in P$ and all $a, b \geq 0$, and $P \cap(-P)=\{\theta\}$, where $\theta$ is the zero element of $E$. A cone $P$ of $E$ determines a partial order $\preceq$ on $E$ by $x \preceq y \Leftrightarrow y-x \in P$ for all $x, y \in X$. For all $x, y \in E$ with $y-x \in$ int $P$, we write $x \ll y$, where int $P$ is the interior of $P$.

Let $P$ be a cone of a topological vector space. $P$ is total order minihedral [29] if, for all upper bounded nonempty total ordered subset $A$ of $E$, sup $A$ exists in $E$. Equivalently, $P$ is total order minihedral if, for all lower bounded nonempty total ordered subset $A$ of $E$, inf $A$ exists in $E$.

Let $E$ be a normed vector space. A cone $P$ of $E$ is continuous $[1,3]$ if, for all subset $A$ of $E$, inf $A$ exists implies $\inf _{x \in A}\|x-\inf A\|=0$, and sup $A$ exists implies $\sup _{x \in A} \| x-$ $\sup A \|=0$. A cone $P$ of $E$ is normal [30] if there exists $N>0$ such that for all $x, y \in P, x \preceq y$ implies $\|x\| \leq N\|y\|$, and the minimal $N$ is called a normal constant of $P$. Equivalently, A cone $P$ of $E$ is normal provided that for all $\left\{x_{n}\right\},\left\{y_{n}\right\},\left\{z_{n}\right\} \subseteq E$ with $x_{n} \preceq y_{n} \preceq z_{n}$ for all $n, x_{n} \rightarrow x$ and $z_{n} \rightarrow x$ imply $y_{n} \rightarrow x$ for some $x \in X$.

Remark 1. A total order minihedral cone $P$ of a normed space $E$ is certainly normal see [29].

Let $X$ be a nonempty set and $P$ a cone of a topological vector space $E$. A cone metric [28] is a mapping $d: X \times X \rightarrow$ $P$ such that for all $x, y, x \in X$,

$(d 1) d(x, y)=\theta$ if and only if $x=y$,

$(d 2) d(x, y)=d(y, x)$,

(d3) $d(x, y) \preceq d(x, z)+d(z, y)$.

A pair $(X, d)$ is called a cone metric space over $P$ if $d: X \times$ $X \rightarrow P$ is a cone metric. Let $(X, d)$ be a cone metric space over a cone $P$ of a topological vector space $E$. A sequence $\left\{x_{n}\right\}$ in $(X, d)$ converges [28] to $x \in X$ (denote $x_{n} \stackrel{d}{\rightarrow} x$ ) if, for all $\varepsilon \in P$ with $\theta \ll \varepsilon$, there exists a positive integer $n_{0}$ such that $d\left(x_{n}, x\right) \ll \varepsilon$ for all $n \geq n_{0}$. A sequence $\left\{x_{n}\right\}$ in $(X, d)$ is Cauchy [28] if, for all $\varepsilon \in P$ with $\theta \ll \varepsilon$, there exists a positive integer $n_{0}$ such that $d\left(x_{n}, x_{m}\right) \ll \varepsilon$ for all $m, n \geq n_{0}$. A cone metric space $(X, d)$ is complete [28] if all Cauchy sequence $\left\{x_{n}\right\}$ in $(X, d)$ converges to a point $x \in X$. A vector functional $\varphi: X \rightarrow E$ is sequentially continuous at some $x \in X$ if $\lim _{n \rightarrow \infty} \varphi\left(x_{n}\right)=\varphi(x)$ for all $\left\{x_{n}\right\} \subseteq X$ such that $x_{n} \stackrel{d}{\rightarrow} x$. If, for all $x \in X, \varphi$ is sequentially continuous at $x$, then $\varphi: X \rightarrow$ $E$ is sequentially continuous.

Remark 2. Let $(X, d)$ be a cone metric space over a normal cone $P$ of a normed vector space $E$ and $\left\{x_{n}\right\}$ a sequence in
$(X, d)$. Then $x_{n} \stackrel{d}{\rightarrow} x$ if and only if $\lim _{n \rightarrow \infty} d\left(x_{n}, x\right)=\theta$, and $\left\{x_{n}\right\}$ is Cauchy if and only if $\lim _{m, n \rightarrow \infty} d\left(x_{n}, x_{m}\right)=\theta$ see [28].

Let $X$ be a nonempty set and $\prec$ a partial order on $X$. For all $x, y \in X$ with $x \prec y$, set $[x,+\infty)=\{z \in X: x \prec z\}$, $(-\infty, x]=\{z \in X: z \prec x\}$, and $[x, y]=\{z \in X: x \prec z \prec y\}$. Let $A$ be a nonempty subset of $X$. A set-valued mapping $T$ : $X \rightarrow 2^{X}$ is increasing on $A$ if, for all $x, y \in A$ with $x \prec y$ and all $u \in T x$, there exists $v \in T y$ such that $u \prec v$. A set-valued mapping $T: X \rightarrow 2^{X}$ is quasi-increasing if, for all $x, y \in A$ with $x \prec y$ and all $v \in T y$, there exists $u \in T x$ such that $u \prec v$. In particular, a single-valued mapping $T: X \rightarrow X$ is increasing on $A$ if, for all $x, y \in A$ with $x \prec y, T x \prec T y$.

A point $x^{*} \in X$ is called a fixed point of a set-valued (resp., single-valued) mapping $T$ if $x^{*} \in T x^{*}$ (resp. $x^{*}=T x^{*}$ ). Let $A$ be a nonempty subset of $X$ and let $x^{*} \in A$ be a fixed point of a mapping T. $x^{*}$ is called a maximal (resp. minimal) fixed point of $T$ in $A$ if for all fixed point $x \in A$ of $T, x^{*} \prec x$ (resp., $x \prec x^{*}$ ) implies $x^{*}=x . x^{*} \in A$ is called a largest (resp., least) fixed point of $T$ in $A$ if, for all fixed point $x \in A$ of $T, x \prec x^{*}$ (resp., $x^{*} \prec x$ ). A largest (resp., least) fixed point of $T$ in $A$ is naturally a maximal (resp., minimal) fixed point in $A$, but the converse may not be true.

\section{Fixed Point Theorems}

In this section, we always assume that the partial order $\prec$ is defined by (1).

Theorem 3. Let $(X, d, \prec)$ be a complete partially ordered cone metric space over a total order minihedral and continuous cone $P$ of a normed vector space E. Let $\varphi: X \rightarrow$ E be a sequentially continuous vector functional and let $T: X \rightarrow 2^{X}$ be a setvalued mapping such that $T x$ is compact for all $x \in X$. Assume that there exists $x_{0} \in X$ such that $\varphi$ is bounded below on $\left[x_{0},+\infty\right), T$ is increasing on $\left[x_{0},+\infty\right)$, and T $x_{0} \cap\left[x_{0},+\infty\right) \neq \emptyset$. Then $T$ has a maximal fixed point $x^{*} \in\left[x_{0},+\infty\right)$.

Proof. Since $P$ is a total order minihedral cone and $E$ is a normed space, then $P$ is a normal cone by Remark 1 . Set

$$
Q_{1}=\left\{x \in\left[x_{0},+\infty\right): T x \cap[x,+\infty) \neq \emptyset\right\} .
$$

Clearly, $Q_{1}$ is nonempty since $x_{0} \in Q_{1}$. Let $\left\{x_{\alpha}\right\}_{\alpha \in \Gamma} \subseteq Q_{1}$ be an increasing chain, where $\Gamma$ is a directed set. Then by (1) we have

$$
d\left(x_{\alpha}, x_{\beta}\right) \preceq \varphi\left(x_{\alpha}\right)-\varphi\left(x_{\beta}\right),
$$

for all $\alpha, \beta \in \Gamma$ with $\alpha \preceq \beta$. This implies that $\left\{\varphi\left(x_{\alpha}\right)\right\}$ is a decreasing chain in $E$. Since $P$ is total order minihedral and $\varphi$ is bounded below on $\left[x_{0},+\infty\right)$, then $\inf _{\alpha \in \Gamma} \varphi\left(x_{\alpha}\right)$ exists in $E$. Moreover, inf $\operatorname{in}_{\alpha \in \Gamma}\left\|\varphi\left(x_{\alpha}\right)-\inf _{\alpha \in \Gamma} \varphi\left(x_{\alpha}\right)\right\|=0$ since $P$ is continuous. Therefore there exists an increasing sequence $\left\{x_{\alpha_{n}}\right\} \subseteq\left\{x_{\alpha}\right\}$ such that $\lim _{n \rightarrow \infty}\left\|\varphi\left(x_{\alpha_{n}}\right)-\inf _{\alpha \in \Gamma} \varphi\left(x_{\alpha}\right)\right\|=0$, that is,

$$
\lim _{n \rightarrow \infty} \varphi\left(x_{\alpha_{n}}\right)=\inf _{\alpha \in \Gamma} \varphi\left(x_{\alpha}\right)
$$


By (1) we have for all $m \in \mathbb{N}$ such that $m \geq n$,

$$
d\left(x_{\alpha_{n}}, x_{\alpha_{m}}\right) \preceq \varphi\left(x_{\alpha_{n}}\right)-\varphi\left(x_{\alpha_{m}}\right) .
$$

Let $n \rightarrow \infty$, by (6) we have $\lim _{m, n \rightarrow \infty}\left[\varphi\left(x_{\alpha_{n}}\right)-\varphi\left(x_{\alpha_{m}}\right)\right]=$ $\theta$ and hence $\lim _{m, n \rightarrow \infty} d\left(x_{\alpha_{n}}, x_{\alpha_{m}}\right)=\theta$ by the normality of $P$. Moreover by Remark 2, $\left\{x_{\alpha_{n}}\right\}$ is a Cauchy sequence in $X$. Therefore by the completeness of $X$, there exists some $\bar{x} \in X$ such that

$$
x_{\alpha_{n}} \stackrel{d}{\longrightarrow} \bar{x}
$$

Note that $\left\{x_{\alpha_{n}}\right\}$ is an increasing sequence of $Q_{1}$, then by (1), we have for all $n$,

$$
d\left(x_{0}, x_{\alpha_{n}}\right) \preceq \varphi\left(x_{0}\right)-\varphi\left(x_{\alpha_{n}}\right),
$$

And, for all $n \geq n_{0}$,

$$
d\left(x_{\alpha_{n_{0}}}, x_{\alpha_{n}}\right) \preceq \varphi\left(x_{\alpha_{n_{0}}}\right)-\varphi\left(x_{\alpha_{n}}\right)
$$

where $n_{0}$ is an arbitrary integer. Let $n \rightarrow \infty$, then by (8) and the continuity of $\varphi$ we have $d\left(x_{0}, \bar{x}\right) \preceq \varphi\left(x_{0}\right)-\varphi(\bar{x})$ and $d\left(x_{\alpha_{n_{0}}}, \bar{x}\right) \preceq \varphi\left(x_{\alpha_{n_{0}}}\right)-\varphi(\bar{x})$, that is, $\bar{x} \in\left[x_{0},+\infty\right)$ and $x_{\alpha_{n_{0}}} \prec \bar{x}$. Moreover the arbitrary property of $n_{0}$ forces that

$$
x_{\alpha_{n}} \prec \bar{x}
$$

for all $n$. By $x_{\alpha_{n}} \in Q_{1}$, there exists $y_{n} \in T x_{\alpha_{n}}$ such that

$$
x_{\alpha_{n}} \prec y_{n}
$$

for all $n$. Since $T$ is increasing on $\left[x_{0},+\infty\right)$, then by (11) and $\bar{x} \in\left[x_{0},+\infty\right)$, there exists $z_{n} \in T \bar{x}$ such that

$$
y_{n} \prec z_{n}
$$

for all $n$. This together with (12) implies that

$$
x_{\alpha_{n}} \prec z_{n}
$$

for all $n$. Note that $T \bar{x}$ is compact, and there exists a subsequence $\left\{z_{n_{k}}\right\} \subseteq\left\{z_{n}\right\}$ and $z \in T \bar{x}$ such that

$$
z_{n_{k}} \longrightarrow z
$$

From (14) we have $x_{\alpha_{n_{k}}} \prec z_{n_{k}}$ for all $n_{k}$ and hence by (1),

$$
d\left(x_{\alpha_{n_{k}}}, z_{n_{k}}\right) \preceq \varphi\left(x_{\alpha_{n_{k}}}\right)-\varphi\left(z_{n_{k}}\right)
$$

for all $n_{k}$. Let $n_{k} \rightarrow \infty$, then by (8), (15), and the continuity of $\varphi$ we have $d(\bar{x}, z) \preceq \varphi(\bar{x})-\varphi(z)$, that is, $\bar{x} \prec z$. This implies that $T \bar{x} \cap[\bar{x},+\infty) \neq \emptyset$ and hence $\bar{x} \in Q_{1}$ by $\bar{x} \in\left[x_{0},+\infty\right)$.

For all $\alpha \in \Gamma$, if there exists some $n_{0}$ such that $x_{\alpha} \prec x_{\alpha_{n_{0}}}$, then $\bar{x}$ is an upper bound of $\left\{x_{\alpha}\right\}$ by (11). Otherwise, there exists some $\beta \in \Gamma$ such that $x_{\alpha_{n}} \prec x_{\beta}$ for all $n$. Thus by (1) we have $\varphi\left(x_{\alpha_{n}}\right)-\varphi\left(x_{\beta}\right) \in P$ for all $n$. Let $n \rightarrow \infty$, by (6) we have $\inf _{\alpha \in \Gamma} \varphi\left(x_{\alpha}\right)-\varphi\left(x_{\beta}\right) \in P$; that is, $\varphi\left(x_{\beta}\right) \preceq \inf _{\alpha \in \Gamma} \varphi\left(x_{\alpha}\right)$. So we have $\varphi\left(x_{\beta}\right)=\inf _{\alpha \in \Gamma} \varphi\left(x_{\alpha}\right)$ and hence $\varphi\left(x_{\beta}\right) \preceq \varphi\left(x_{\alpha}\right)$ for all $\alpha \in \Gamma$. Note that $\left\{\varphi\left(x_{\alpha}\right)\right\}_{\alpha \in \Gamma}$ is a decreasing chain, then $\beta \geq \alpha$ for all $\alpha \in \Gamma$. Moreover $x_{\alpha} \prec x_{\beta}$ for all $\alpha \in \Gamma$ since $\left\{x_{\alpha}\right\}_{\alpha \in \Gamma}$ is an increasing chain. Hence $\left\{x_{\alpha}\right\}_{\alpha \in \Gamma}$ has an upper bound in $Q_{1}$. By Zorn's lemma, $\left(Q_{1}, \prec\right)$ has a maximal element $x^{*}$; that is, for all $x \in Q_{1}, x^{*} \prec x$ implies $x=x^{*}$. By $x^{*} \in Q_{1}$, there exists $y^{*} \in T x^{*}$ such that $x^{*} \prec y^{*}$. Moreover by the increasing property of $T$ on $\left[x_{0},+\infty\right)$, there exists $z^{*} \in T y^{*}$ such that $y^{*} \prec z^{*}$. Thus we have $x^{*} \prec z^{*}$ by $x^{*} \prec y^{*}$. This indicates $z^{*} \in T x^{*} \cap\left[x^{*},+\infty\right)$ and hence $z^{*} \in Q_{1}$. Finally the maximality of $x^{*}$ in $Q_{1}$ forces that $x^{*}=z^{*} \in T x^{*}$; that is, $x^{*}$ is a maximal fixed point of $T$ in $\left[x_{0},+\infty\right)$. The proof is complete.

Theorem 4. Let $(X, d, \prec)$ be a complete partially ordered cone metric space over a total order minihedral and continuous cone $P$ of a normed vector space E. Let $\varphi: X \rightarrow E$ be a sequentially continuous vector functional and $T: X \rightarrow 2^{X}$ be a set-valued mapping such that $T x$ is compact for all $x \in X$. Assume that there exists $y_{0} \in X$ such that $\varphi$ is bounded above on $\left(-\infty, y_{0}\right]$, $T$ is quasi-increasing on $\left(-\infty, y_{0}\right]$, and $T y_{0} \cap\left(-\infty, y_{0}\right] \neq \emptyset$. Then $T$ has a minimal fixed point $x_{*} \in\left(-\infty, y_{0}\right]$.

Proof. Set

$$
Q_{2}=\left\{x \in\left(-\infty, y_{0}\right]: T x \cap(-\infty, x] \neq \emptyset\right\}
$$

Clearly, $Q_{2} \neq \emptyset$. By the same method used in the proof of Theorem 3, we can prove that $\left(Q_{2}, \prec\right)$ has a minimal element $x_{*}$ which is also a minimal fixed point of $T$ in $\left(-\infty, y_{0}\right]$. The proof is complete.

Remark 5. If $T: X \rightarrow X$ is a single-valued mapping, then $T x$ is naturally compact for all $x \in X$. Hence both of Theorems 3 and 4 are still valid for a single-valued mapping.

In particular when $T$ is a single-valued mapping, we have the following further results.

Theorem 6. Let $(X, d, \prec)$ be a complete partially ordered cone metric space over a total order minihedral and continuous cone $P$ of a normed vector space E. Let $\varphi: X \rightarrow E$ be a sequentially continuous vector functional and let $T: X \rightarrow X$ be a singlevalued mapping. Assume that there exists $x_{0} \in X$ such that $\varphi$ is bounded below on $\left[x_{0},+\infty\right), T$ is increasing on $\left[x_{0},+\infty\right)$, and $x_{0} \prec T x_{0}$. Then $T$ has a maximal fixed point $x^{*}$ and a least fixed point $x_{*}$ in $\left[x_{0},+\infty\right)$ such that $x_{*} \prec x^{*}$.

Proof. By Theorem 3 and Remark 5, T has a maximal fixed point $x^{*} \in\left[x_{0},+\infty\right)$ and hence $F=\left\{x \in\left[x_{0},+\infty\right): x=\right.$ $T x\} \neq \emptyset$. Set

$$
S=\left\{I=[x,+\infty): x \in\left[x_{0},+\infty\right), x \prec T x, F \subseteq I\right\} .
$$

Clearly, $\left[x_{0},+\infty\right) \in S$ and hence $S \neq \emptyset$. Define a relation $\sqsubseteq$ on $S$ by

$$
I_{1} \sqsubseteq I_{2} \Longleftrightarrow I_{1} \subseteq I_{2}
$$

for all $I_{1}, I_{2} \in S$, then it is easy to check that $\sqsubseteq$ is a partial order on $S$. 
Let $\left\{I_{\alpha}\right\}_{\alpha \in \Gamma}$ be a decreasing chain of $S$, where $I_{\alpha}=$ $\left[x_{\alpha},+\infty\right)$. From (1), (18), and (19) we find that $\left\{x_{\alpha}\right\}_{\alpha \in \Gamma}$ is an increasing chain of $M$, where

$$
M=\left\{x \in\left[x_{0},+\infty\right): x \prec T x, F \subseteq[x,+\infty)\right\} .
$$

Set $\bar{Q}_{1}=\left\{x \in\left[x_{0},+\infty\right): x \prec T x\right\}$. Clearly, $M \subseteq \bar{Q}_{1}$. Following the proof of Theorem 3, there exists $\bar{x} \in \bar{Q}_{1}$ and an increasing sequence $\left\{x_{\alpha_{n}}\right\} \subseteq\left\{x_{\alpha}\right\}$ satisfying (6) such that (8) and (11) are satisfied. From $x_{\alpha_{n}} \in M$ we have that $x_{\alpha_{n}} \prec x$ for all $x \in F$ and all $n$. Thus the increasing property of $T$ on $\left[x_{0},+\infty\right)$ implies that, for all $x \in F$ and all $n$,

$$
x_{\alpha_{n}} \prec T x_{\alpha_{n}} \prec T x=x,
$$

and hence by (1),

$$
d\left(x_{\alpha_{n}}, x\right) \preceq \varphi\left(x_{\alpha_{n}}\right)-\varphi(x),
$$

for all $x \in F$ and all $n$. Let $n \rightarrow \infty$, then by (8) and the continuity of $\varphi$ we have $d(\bar{x}, x) \preceq \varphi(\bar{x})-\varphi(x)$; that is,

$$
\bar{x} \prec x,
$$

for all $x \in F$. This together with $\bar{x} \in \bar{Q}_{1}$ implies $\bar{x} \in M$. Then in analogy to the proof of Theorem 3 , by (6), (8), and $\bar{x} \in M$ we can prove $\left\{x_{\alpha}\right\}_{\alpha \in \Gamma}$ has an upper bound $\hat{x} \in M$. By (18), we have $[\hat{x},+\infty) \in S$. Note that $\widehat{x}$ is an upper bound of $\left\{x_{\alpha}\right\}_{\alpha \in \Gamma}$ in $M$, then $[\widehat{x},+\infty) \subseteq I_{\alpha}$ for all $\alpha \in \Gamma$ and hence by (19),

$$
[\widehat{x},+\infty) \sqsubseteq I_{\alpha}
$$

for all $\alpha \in \Gamma$. This means $[\widehat{x},+\infty)$ is a lower bound of $\left\{I_{\alpha}\right\}_{\alpha \in \Gamma}$ in $S$. By Zorn's lemma, $(S, \sqsubseteq)$ has a minimal element; denote it by $I^{*}=\left[x_{*},+\infty\right)$. By (18) we have $x_{0} \prec x_{*} \prec T x_{*}$ and

$$
x_{*} \prec x,
$$

for all $x \in F$. By the increasing property of $T$, we have $x_{0} \prec$ $x_{*} \prec T x_{*} \prec T\left(T x_{*}\right)$ and $T x_{*} \prec T x=x$ for all $x \in F$, which implies $\left[T x_{*},+\infty\right) \in S$ and $\left[T x_{*},+\infty\right) \subseteq I^{*}$. Moreover by (19), $\left[T x_{*},+\infty\right) \sqsubseteq I^{*}$. The minimality of $I^{*}$ in $S$ forces that $\left[T x_{*},+\infty\right)=I^{*}$ and so we have $x_{*}=T x_{*}$. Finally by (25), $x_{*}$ is a least fixed point of $T$ in $\left[x_{0},+\infty\right)$ and $x_{*} \prec x^{*}$. The proof is complete.

Theorem 7. Let $(X, d, \prec)$ be a complete partially ordered cone metric space over a total order minihedral and continuous cone $P$ of a normed vector space E. Let $\varphi: X \rightarrow E$ be a sequentially continuous vector functional and let $T: X \rightarrow X$ be a singlevalued mapping. Assume that there exists $x_{0} \in X$ such that $\varphi$ is bounded above on $\left(-\infty, y_{0}\right], T$ is increasing on $\left(-\infty, y_{0}\right]$, and $T y_{0} \prec y_{0}$. Then $T$ has a minimal fixed point $x_{*}$ and a largest fixed point in $x^{*}$ in $\left(-\infty, x_{0}\right]$ such that $x_{*} \prec x^{*}$.

Proof. By Theorem 4 and Remark 5, $T$ has a minimal fixed point in $x_{*} \in\left(-\infty, y_{0}\right]$. Set

$$
\bar{S}=\left\{J=(-\infty, x]: x \in\left(-\infty, y_{0}\right], T x \prec x, F \subseteq J\right\} .
$$

Define a relation $\sqsubseteq \bar{S}$ on $\bar{S}$ as follows:

$$
J_{1} \sqsubseteq \bar{S} J_{2} \Longleftrightarrow J_{1} \subseteq J_{2},
$$

for all $J_{1}, J_{2} \in \bar{S}$, then $\complement_{\bar{S}}$ is a partial order on $\bar{S}$. In an analogy to the proof of Theorem 4 , we can prove $\left(\bar{S}, \complement_{\bar{S}}\right)$ has a minimal element $\left(-\infty, x^{*}\right]$ and $x^{*}$ is a largest fixed point of $T$ in $\left(-\infty, y_{0}\right]$. The proof is complete.

Theorem 8. Let $(X, d, \prec)$ be a complete partially ordered cone metric space over a total order minihedral and continuous cone $P$ of a normed vector space E. Let $\varphi: X \rightarrow E$ be a sequentially continuous mapping and let $T: X \rightarrow X$ be a single-valued mapping. Assume that there exists $x_{0}, y_{0} \in X$ with $x_{0} \prec y_{0}$ such that $T$ is increasing on $\left[x_{0}, y_{0}\right]$ and $x_{0} \prec T x_{0}, T y_{0} \prec y_{0}$. Then $T$ has a largest fixed point $x^{*}$ and a least fixed point $x_{*}$ in $\left[x_{0}, y_{0}\right]$ such that $x_{*} \prec x^{*}$.

Proof. For all $x \in\left[x_{0}, y_{0}\right]$, by (1) we have $\varphi\left(y_{0}\right) \preceq \varphi(x) \prec$ $\varphi\left(x_{0}\right)$; that is, $\varphi$ is bounded on $\left[x_{0}, y_{0}\right]$. In an analogy to the proof of Theorem 3, we can prove $T$ has a maximal fixed point and a minimal fixed point in $\left[x_{0}, y_{0}\right]$ by investigating the existence of maximal element and minimal element, respectively, in $D_{1}=\left\{x \in\left[x_{0}, y_{0}\right]: x \prec T x\right\}$ and $D_{2}=$ $\left\{x \in\left[x_{0}, y_{0}\right]: T x \prec x\right\}$. Let

$$
\begin{aligned}
& S_{1}=\left\{I=\left[x, y_{0}\right]: x \in\left[x_{0}, y_{0}\right], x \prec T x, G \subseteq I\right\}, \\
& S_{2}=\left\{J=\left[x_{0}, x\right]: x \in\left[x_{0}, y_{0}\right], T x \prec x, G \subseteq J\right\},
\end{aligned}
$$

where $G=\left\{x \in\left[x_{0}, y_{0}\right]: T x=x\right\}$ is nonempty. Define $\sqsubseteq_{1}$ on $S_{1}$ and $\sqsubseteq_{2}$ on $S_{2}$, respectively, by

$$
\begin{aligned}
& I_{1} \sqsubseteq_{1} I_{2} \Longleftrightarrow I_{1} \subseteq I_{2}, \quad \forall I_{1}, I_{2} \in S_{1}, \\
& J_{1} \sqsubseteq_{2} J_{2} \Longleftrightarrow J_{1} \subseteq J_{2}, \quad \forall J_{1}, J_{2} \in S_{2},
\end{aligned}
$$

then it is easy to check that $\sqsubseteq_{1}$ and $\sqsubseteq_{2}$ are partial orders on $S_{1}$ and $S_{2}$, respectively. In an analogy to the proof of Theorem 4 , we can prove $\left(S_{1}, \sqsubseteq_{1}\right)$ has a minimal element $I_{*}=\left[x_{*}, y_{0}\right]$ and $\left(S_{2}, \sqsubseteq_{2}\right)$ has a minimal element $J_{*}=\left[x_{0}, y^{*}\right]$. By the definitions of $S_{1}$ and $S_{2}$, we have $x_{*}, y^{*} \in\left[x_{0}, y_{0}\right]$,

$$
\begin{gathered}
x_{*} \prec x \prec y^{*}, \\
x_{*} \prec T x_{*} \prec T y^{*} \prec y^{*} .
\end{gathered}
$$

Moreover by (30) and the increasing property of $T$ on $\left[x_{0}, y_{0}\right]$, for all $x \in G$, we have

$$
x_{0} \prec T x_{0} \prec T x_{*} \prec x \prec T y^{*} \prec T y_{0} \prec y_{0},
$$

and so by (31),

$$
x_{*} \prec T x_{*} \prec T\left(T x_{*}\right) \prec T\left(T y^{*}\right) \prec T y^{*} \prec y^{*} .
$$

From (32) and (33) we have that $\left[T x_{*}, y_{0}\right] \in S_{1},\left[x_{0}, T y^{*}\right] \in$ $S_{2}$, and

$$
\left[T x_{*}, y_{0}\right] \sqsubseteq_{1} I_{*}, \quad\left[x_{0}, T y^{*}\right] \sqsubseteq_{2} J_{*},
$$

which implies $\left[T x_{*}, y_{0}\right]=I_{*}$ and $\left[x_{0}, T y^{*}\right]=J_{*}$ by the minimality of $I_{*}$ and $J_{*}$. This means that $T x_{*}=x_{*}$ and $T y^{*}=$ $y^{*}$. Hence $x_{*}$ is the least fixed point and $y^{*}$ is the largest fixed point of $T$ in $\left[x_{0}, y_{0}\right]$ by (31). The proof is complete. 
Remark 9. Theorems 3-8 are extensions of [4, Theorems 3 and 4] and [2, Theorems 3, 4, and 5] to the case of cone metric spaces. It is worth mentioning that in Theorems 4,7 , and 8 , not only the existence of maximal and minimal fixed points but also the existence of largest and least fixed points is obtained. Therefore Theorems 4, 7, and 8 are still new even in the case of metric space and hence they indeed improve [2, Theorems 3, 4, and 6].

Now we give an example to demonstrate Theorem 3.

Example 10. Let $X=\{1,2,3,4\}, E=\mathbb{R}^{2}$ with the norm $\|u\|=$ $\sqrt{u_{1}^{2}+u_{2}^{2}}$ for all $u=\left(u_{1}, u_{2}\right) \in \mathbb{R}^{2}$ and $P=\mathbb{R}_{+}^{2}$. Clearly, $P$ is a strongly minihedral and continuous cone of $E$. Define a mapping $d: \mathbb{R} \times \mathbb{R} \rightarrow P$ by

$$
d(x, y)=\left(|x-y|,|x-y|^{1 / 2}\right), \quad \forall x, y \in \mathbb{R},
$$

then $(\mathbb{R}, d)$ is a complete cone metric space over $P$ and hence $(X, d)$ is a complete cone metric subspace of $(\mathbb{R}, d)$. Define a vector functional $\varphi:[1,+\infty) \rightarrow E$ by

$$
\varphi(x)=\left(\frac{6}{x}, \frac{3 \sqrt{2}+2 \sqrt{3}}{\sqrt{x}}\right),
$$

for all $x \in[1,+\infty)$. For arbitrary $x \in[1,+\infty)$, let $\left\{x_{n}\right\} \subseteq$ $[1,+\infty)$ be a sequence such that $x_{n} \stackrel{d}{\rightarrow} x$, then $x_{n} \stackrel{|\cdot|}{\rightarrow} x$ and hence $\left\|\varphi\left(x_{n}\right)-\varphi(x)\right\| \rightarrow 0$, that is, $\lim _{n \rightarrow \infty} \varphi\left(x_{n}\right)=\varphi(x)$. This means that $\varphi:[1,+\infty) \rightarrow E$ is sequentially continuous; in particular, $\varphi: X \rightarrow E$ is sequentially continuous. From (35) and (36) it is easy to check that

$$
\begin{aligned}
& 1 \prec 1, \quad 1 \prec 2, \quad 1 \prec 3, \quad 1 \prec 4, \\
& \quad 2 \prec 2, \quad 2 \prec 3, \quad 2 \prec 4, \\
& 3 \prec 3, \quad 3 k 4, \quad 4 \prec 4, \quad 4 k 3,
\end{aligned}
$$

where $\prec$ is the partial order defined by (1). Let $T: X \rightarrow 2^{X}$ be a set-valued mapping such that

$$
\begin{array}{cc}
T 1=\{3,4\}, & T 2=\{1,3\}, \\
T 3=\{1,2,3,4\}, & T 4=\{1,2,3\} .
\end{array}
$$

Fix $x_{0}=2$, then $\left[x_{0},+\infty\right)=\{x \in X: 2 \prec x\}=\{2,3,4\}$ by (37), and so $T x_{0} \cap\left[x_{0},+\infty\right)=\{3\} \neq \emptyset$. For $x, y \in\left[x_{0},+\infty\right)$, if $x \prec y$ and $x \neq y$, then we have only two cases: $x=2 \prec 3=y$ and $x=2 \prec 4=y$ by (37). Fix $x=2$ and $y=3$, for all $u \in T x$, there exists $v=3,4 \in T y$ such that $u \prec v$. Fix $x=2$ and $y=4$, for all $u \in T x$, there exists $v=3 \in T y$ such that $u \prec v$. This means that $T: X \rightarrow 2^{X}$ is increasing on $\left[x_{0},+\infty\right)$. Therefore all the conditions of Theorem 3 are satisfied and hence $T$ has a fixed point $3 \in\left[x_{0},+\infty\right)$.

Fix $x=4$; for all $y \in T 4$, we have $x=4 \nless y$ by (37); that is, (2) is not satisfied. Therefore the existence of fixed points could not be obtained by generalized Caristi's fixed point theorems in cone metric spaces of $[1,3]$.

\section{Acknowledgments}

The work was supported by Natural Science Foundation of China (11161022), Natural Science Foundation of Jiangxi Province (20114BAB211006, 20122BAB201015), Educational Department of Jiangxi Province (GJJ12280), and Program for Excellent Youth Talents of JXUFE (201201). The authors are grateful to the editor and referees for their critical suggestions led to the improvement of the presentation of the work.

\section{References}

[1] R. P. Agarwal and M. A. Khamsi, "Extension of Caristi's fixed point theorem to vector valued metric spaces," Nonlinear Analysis: Theory, Methods \& Applications, vol. 74, no. 1, pp. 141145, 2011.

[2] J. Caristi, "Fixed point theorems for mappings satisfying inwardness conditions," Transactions of the American Mathematical Society, vol. 215, pp. 241-251, 1976.

[3] S. H. Cho, J. S. Bae, and K. S. Na, "Fixed point theorems for multivalued contractive mappings and multivalued Caristi type mappings in cone metric spaces," Fixed Point Theory and Applications, vol. 2012, article 133, 2012.

[4] W. A. Kirk and J. Caristi, "Mappings theorems in metric and Banach spaces," Bulletin de l'Académie Polonaise des Sciences, vol. 23, no. 8, pp. 891-894, 1975.

[5] W. A. Kirk, "Caristi's fixed point theorem and metric convexity," Colloquium Mathematicum, vol. 36, no. 1, pp. 81-86, 1976.

[6] J. R. Jachymski, "Caristi's fixed point theorem and selections of set-valued contractions," Journal of Mathematical Analysis and Applications, vol. 227, no. 1, pp. 55-67, 1998.

[7] J. S. Bae, "Fixed point theorems for weakly contractive multivalued maps," Journal of Mathematical Analysis and Applications, vol. 284, no. 2, pp. 690-697, 2003.

[8] T. Suzuki, "Generalized Caristi's fixed point theorems by Bae and others," Journal of Mathematical Analysis and Applications, vol. 302, no. 2, pp. 502-508, 2005.

[9] Y. Q. Feng and S. Y. Liu, "Fixed point theorems for multi-valued contractive mappings and multi-valued Caristi type mappings," Journal of Mathematical Analysis and Applications, vol. 317, no. 1, pp. 103-112, 2006.

[10] M. A. Khamsi, "Remarks on Caristi's fixed point theorem," Nonlinear Analysis: Theory, Methods \& Applications, vol. 71, no. 1-2, pp. 227-231, 2009.

[11] Z. Li, "Remarks on Caristi's fixed point theorem and Kirk's problem," Nonlinear Analysis: Theory, Methods \& Applications, vol. 73, no. 12, pp. 3751-3755, 2010.

[12] Z. Li and S. Jiang, "Maximal and minimal point theorems and Caristi's fixed point theorem," Fixed Point Theory and Applications, vol. 2011, article 103, 2011.

[13] I. Altun and V. Rakočević, "Ordered cone metric spaces and fixed point results," Computers \& Mathematics with Applications, vol. 60, no. 5, pp. 1145-1151, 2010.

[14] H. K. Nashine, Z. Kadelburg, and S. Radenović, "Coincidence and fixed point results in ordered G-cone metric spaces," Mathematical and Computer Modelling, vol. 57, no. 3-4, pp. 701709, 2013.

[15] D. R. Kurepa, “Tableaux ramifiés d'ensembles, Espaces pseudodistanciés," Comptes Rendus de l'Académie des Sciences, vol. 198, pp. 1563-1565, 1934. 
[16] P. P. Zabrejko, " $K$-metric and $K$-normed linear spaces: survey," Collectanea Mathematica, vol. 48, no. 4-6, pp. 825-859, 1997.

[17] Z. Kadelburg, S. Radenović, and V. Rakočević, "A note on the equivalence of some metric and cone metric fixed point results," Applied Mathematics Letters, vol. 24, no. 3, pp. 370-374, 2011.

[18] S. Janković, Z. Kadelburg, and S. Radenović, "On cone metric spaces: a survey," Nonlinear Analysis: Theory, Methods \& Applications, vol. 74, no. 7, pp. 2591-2601, 2011.

[19] Z. Kadelburg, M. Pavlović, and S. Radenović, "Common fixed point theorems for ordered contractions and quasicontractions in ordered cone metric spaces," Computers \& Mathematics with Applications, vol. 59, no. 9, pp. 3148-3159, 2010.

[20] M. A. Alghamdi, S. H. Alnafei, S. Radenović, and N. Shahzad, "Fixed point theorems for convex contraction mappings on cone metric spaces," Mathematical and Computer Modelling, vol. 54, no. 9-10, pp. 2020-2026, 2011.

[21] I. Arandjelović, Z. Kadelburg, and S. Radenović, "Boyd-Wongtype common fixed point results in cone metric spaces," Applied Mathematics and Computation, vol. 217, no. 17, pp. 7167-7171, 2011.

[22] S. H. Alnafei, S. Radenović, and N. Shahzad, "Fixed point theorems for mappings with convex diminishing diameters on cone metric spaces," Applied Mathematics Letters, vol. 24, no. 12, pp. 2162-2166, 2011.

[23] W. Shatanawi, V. Ć. Rajić, S. Radenović, and A. Al-Rawashdeh, "Mizoguchi-Takahashi-type theorems in tvs-cone metric spaces," Fixed Point Theory and Applications, vol. 2012, article 106, 2012.

[24] S. Radenović and Z. Kadelburg, "Quasi-contractions on symmetric and cone symmetric spaces," Banach Journal of Mathematical Analysis, vol. 5, no. 1, pp. 38-50, 2011.

[25] X. Zhang, "Fixed point theorems of monotone mappings and coupled fixed point theorems of mixed monotone mappings in ordered metric spaces," Acta Mathematica Sinica, vol. 44, no. 4, pp. 641-646, 2001 (Chinese).

[26] X. Zhang, "Fixed point theorems of multivalued monotone mappings in ordered metric spaces," Applied Mathematics Letters, vol. 23, no. 3, pp. 235-240, 2010.

[27] Z. Li, "Fixed point theorems in partially ordered complete metric spaces," Mathematical and Computer Modelling, vol. 54, no. 1-2, pp. 69-72, 2011.

[28] L. G. Huang and X. Zhang, "Cone metric spaces and fixed point theorems of contractive mappings," Journal of Mathematical Analysis and Applications, vol. 332, no. 2, pp. 1468-1476, 2007.

[29] Y. H. Du, "Total order minihedral cones," Journal of Systems Science and Mathematical Sciences, vol. 8, no. 1, pp. 19-24, 1988.

[30] K. Deimling, Nonlinear Functional Analysis, Springer, Berlin, Germany, 1985. 


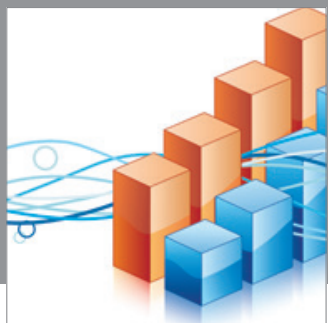

Advances in

Operations Research

mansans

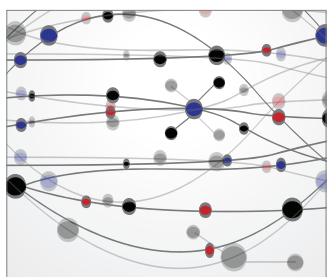

The Scientific World Journal
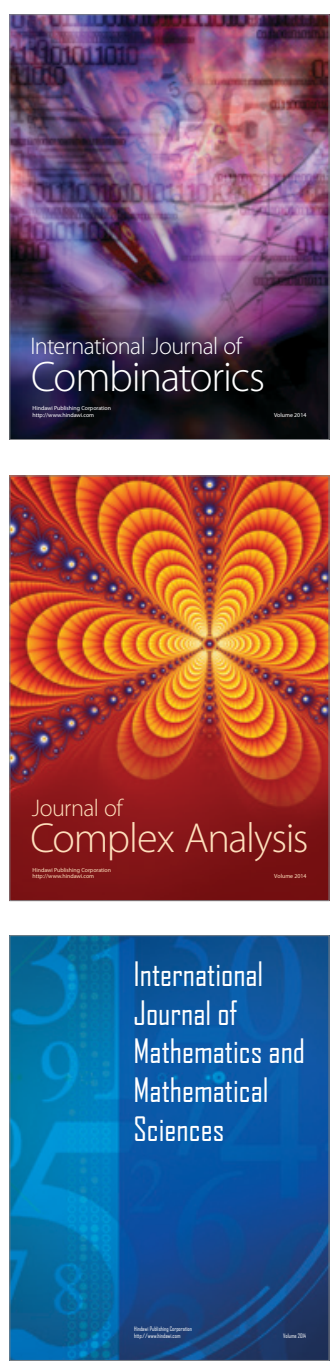
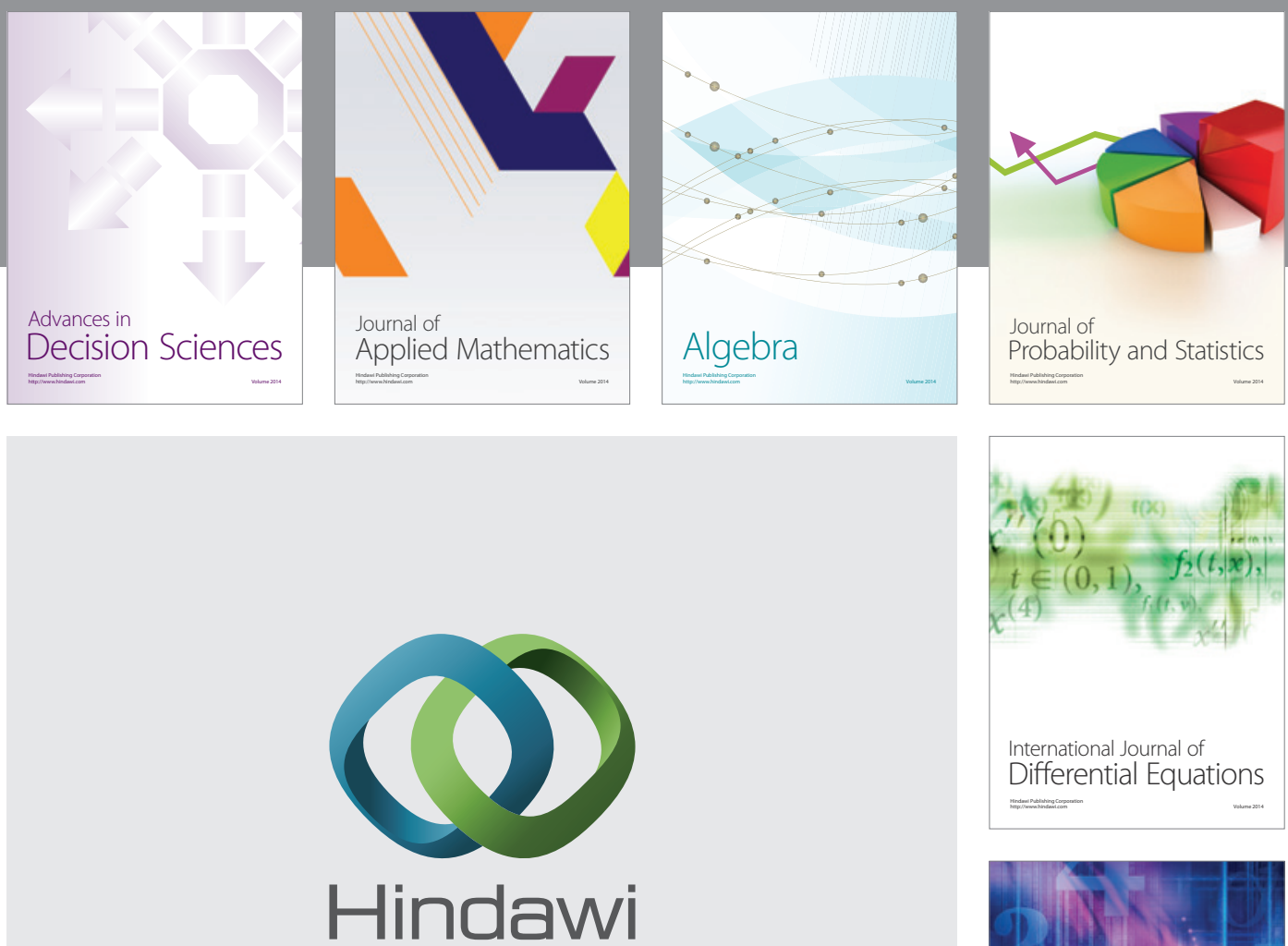

Submit your manuscripts at http://www.hindawi.com
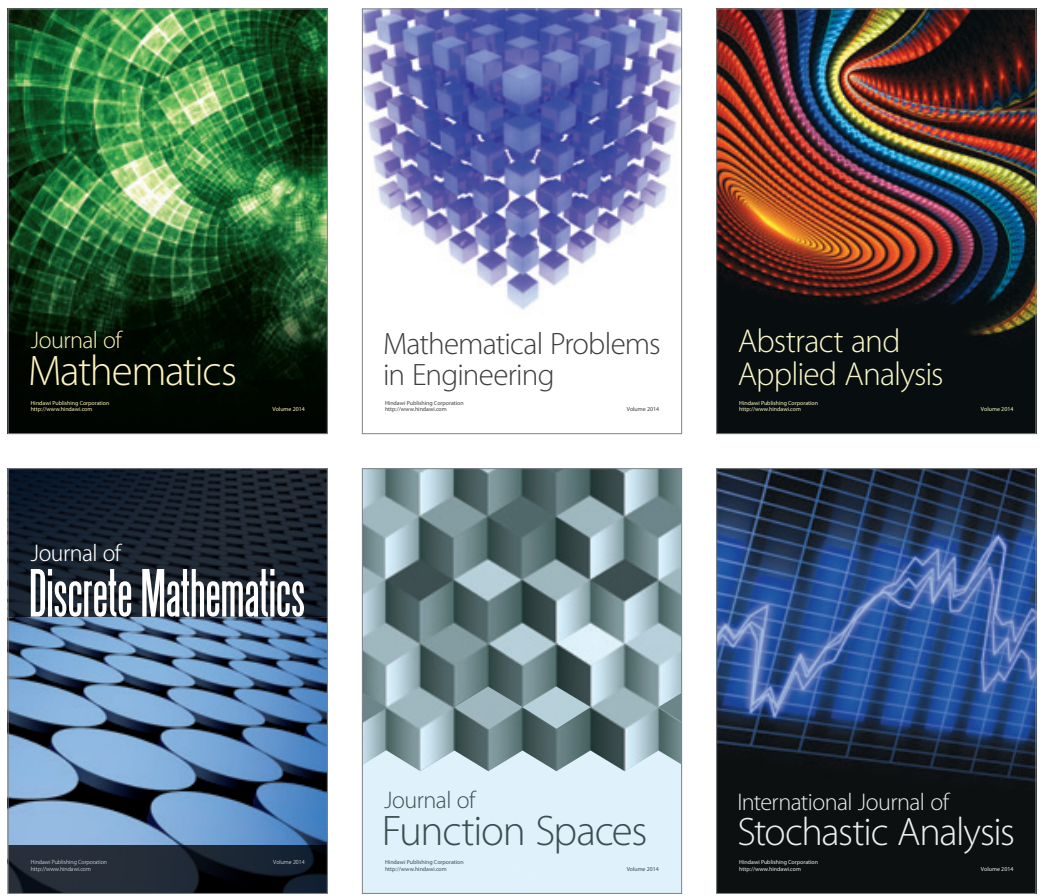

Journal of

Function Spaces

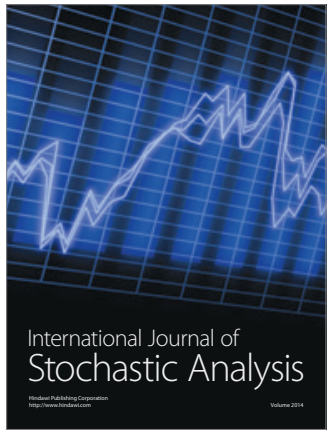

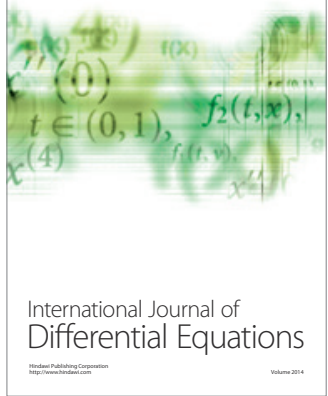
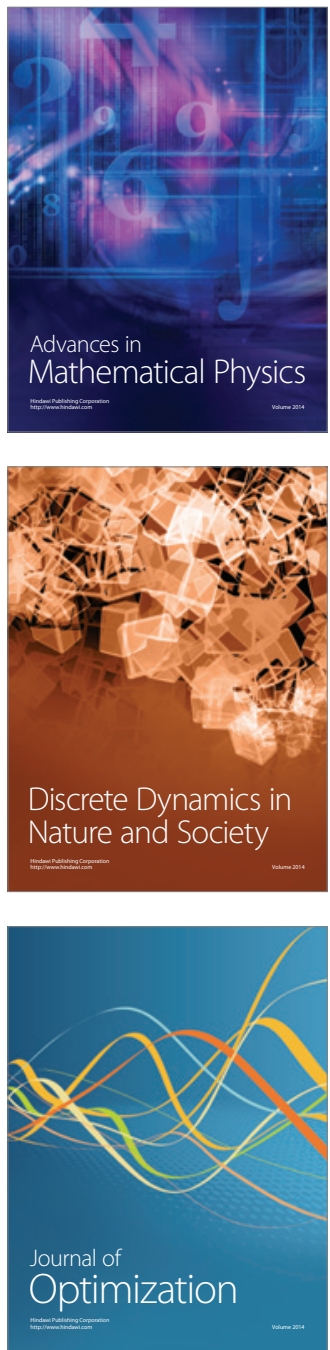